\title{
Editorial
}

Int Neurourol J 2011;15:59-60

doi: 10.5213/inj.2011.15.2.59

pISSN 2093-4777 · eISSN 2093-6931

\section{New Insights into the Mechanism of the Down-Regulation of Mast Cells in the Treatment of Interstitial Cystitis: Possible Role of Siglecs}

Tack Lee

Editor-in-Chief

Department of Urology, Inha University Hospital, Inha University School of Medicine, Incheon, Korea

E-mail: lt11@inha.ac.kr

Interstitial cystitis (IC) is a severely debilitating, still puzzling, heterogeneous syndrome affecting the urinary bladder that is associated with pelvic pain and voiding symptoms of urgency, frequency, and nocturia [1]. Despite many continuing efforts to ascertain the etiology and pathophysiology of IC, our knowledge remains fragmentary and has failed to provide useful insights into effective treatments. Various treatment modalities are currently available to treat patients with IC, but none is particularly effective. New effective treatments will not be available until the etiology of the disease is better understood and the core factors involved in its pathophysiology are better identified. Thus, this is a good time to refresh and upgrade our information related to IC from the view of fields outside of urology.

The idea that IC is an inflammatory response induced by toxic, allergic, or immunologic pathogens in the urine is still the most convincing theory on the pathogenesis of the disease. Furthermore, most studies in humans and animals have focused on the central role of mast cells in IC, although ongoing controversy exists over the detailed mechanisms [2]. Allergic inflammatory responses in urologic organs share many characteristic biochemical and cellular features with organs beyond urology, in which the mast cell is also considered to be one of the key effector cells of the allergic reaction. Recently, a new therapeutic ap- proach based on new biological insights has emerged to better control mast cell function. For example, activation of inhibitory receptors on mast cells by use of antibodies or small molecules for their selective targeting has been shown to be clinically effective. Furthermore, at least in immune systems, regulation of these bindings may result in subsequent down-regulation of cell function.

The vertebrate immune system maintains normal immune cell homeostasis by modulating immune responses through the interplay of activation and inhibition. The cell system of inhibitory signaling is especially crucial for this balancing. Loss of inhibitory signaling may result in autoimmune disease. Since the sialic-acid-binding immunoglobulin-like lectins (Siglecs) were identified for the first time as markers of leukemia and lymphoma 30 years ago [3], they have become known as important inhibitory regulators of immune cell signaling. Their structural features include an N-terminal carbohydrate-binding (lectin) domain that binds sialic acid and immunoreceptor tyrosinebased inhibitory motifs (ITIMs) in their cytoplasmic domains. The ITIMs are responsible for their inhibitory capacity [4]. Siglec-8, the eighth member identified, was discovered as part of an effort to find new eosinophil surface proteins. Subsequent studies showed that mast cells also selectively express Siglec- 8 


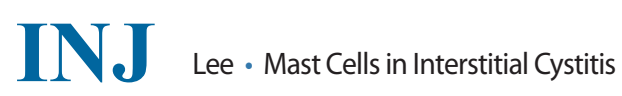

and showed an inhibition of degranulation without apoptosis by crosslinking of Siglec-8, whereas on eosinophils, Siglec- 8 engagement resulted in apoptosis [3].

Thus, the selective targeting of particular Siglecs such as Siglec- 8 on the mast cell could one day be useful in the armamentarium for the treatment of IC. Such targeting could result in the down-regulation of mast cells where overproduction of mast cells is occurring.

The article by Park and Bochner [5] in this issue of the journal will be recognized as a valuable work in the IC field that initiates future research into the inhibitory control of mast cells via manipulation of Siglecs.

\section{REFERENCES}

1. Oberpenning F, van Ophoven A, Hertle L. Interstitial cystitis: an update. Curr Opin Urol 2002;12:321-32.

2. Theoharides TC, Kempuraj D, Sant GR. Mast cell involvement in interstitial cystitis: a review of human and experimental evidence. Urology 2001;57(6 Suppl 1):47-55.

3. Bochner BS. Siglec-8 on human eosinophils and mast cells, and Siglec-F on murine eosinophils, are functionally related inhibitory receptors. Clin Exp Allergy 2009;39:317-24.

4. O'Reilly MK, Paulson JC. Siglecs as targets for therapy in immunecell-mediated disease. Trends Pharmacol Sci 2009;30:240-8.

5. Park CS, Bochner BS. Potential targeting of siglecs, mast cell inhibitory receptors, in interstitial cystitis. Int Neurourol J 2011;15:61-3. 